\section{Comportamento sedentário e associação com multimorbidade e padrões de multimorbidade em idosos brasileiros: dados da Pesquisa Nacional de Saúde de 2019}

\author{
Sedentary behavior and association with \\ multimorbidity and patterns of multimorbidity in \\ elderly Brazilians: data from the Brazilian National \\ Health Survey, 2019
}

\section{Comportamiento sedentario y su asociación con la multimorbilidad y patrones de multimorbilidad en ancianos brasileños: datos de la Encuesta} Nacional de Salud de 2019
Letícia Martins Cândido 1

Kátia Jakovljevic Pudla Wagner 1

Maria Eduarda da Costa 1

Eloísa Pavesi 1

Núbia Carelli Pereira de Avelar 1

Ana Lúcia Danielewicz 1

\title{
Resumo
}

O comportamento sedentário emerge como um importante determinante da saúde da pessoa idosa, no entanto, sua relação com a multimorbidade e seus padrões de acometimento em estudos epidemiológicos têm sido pouco explorados na população brasileira. Tais associações poderão auxiliar na elaboração de políticas públicas visando à modificação desse comportamento. Assim, o objetivo deste estudo foi avaliar a associação entre comportamento sedentário com multimorbidade e seus padrões em idosos brasileiros. Estudo transversal, com 43.554 idosos da Pesquisa Nacional de Saúde de 2019. O comportamento sedentário autorreferido foi categorizado em $<3$; 3-6; e $>6$ horas por dia. A presença de multimorbidade e seus padrões foram analisados pelo autorrelato da coexistência de duas ou mais doenças crônicas, sendo que os três padrões estabelecidos consideraram as doenças com características clínicas similares: (1) cardiopulmonar; (2) vascular-metabólico; e (3) mental-musculoesquelético. As associações foram realizadas pela regressão logística binária. Os idosos que despendiam 3-6 horas/dia em comportamento sedentário tiveram maiores chances $(O R=1,39$; IC95\%: 1,29; 1,50) de apresentar multimorbidade, padrão vascular-metabólico $(O R=1,39$; IC95\%: 1,29; 1,50) e mentalmusculoesquelético $(O R=1,11$; IC95\%: 1,00; 1,24). Quando o comportamento sedentário foi $>6$ horas/dia, houve maiores chances de multimorbidade $(O R=1,58$; IC95\%: 1,43; 1,74) e dos padrões cardiopulmonar $(O R=1,73$; IC95\%: 1,33; 2,27), vascular-metabólico $(O R=1,49$; IC95\%: 1,35; 1,64) e mental-musculoesquelético $(O R=1,15$; IC95\%: 1,$01 ; 1,31)$, quando comparados àqueles que ficavam até 3 horas/dia. Dessa forma, evidenciou-se que tempos elevados em comportamento sedentário são fatores de risco relevantes para a ocorrência de multimorbidade e seus padrões em idosos.

Idoso; Multimorbidade; Comportamento Sedentário; Fatores de Risco

\author{
Correspondência \\ L. M. Cândido \\ Universidade Federal de Santa Catarina. \\ Rod. Governador Jorge Lacerda 3201, Araranguá, SC 88906- \\ 072, Brasil. \\ leticia.candido96@gmail.com
}

1 Universidade Federal de Santa Catarina, Araranguá, Brasil. 


\section{Introdução}

No Brasil, de forma similar ao que ocorre mundialmente, tem-se observado um aumento expressivo da população idosa (maior de 60 anos), que em números absolutos passou de 3 para 32,9 milhões entre os anos de 1960 e 2019 1. Estima-se que, em 2050, em nível mundial, 1 em cada 6 indivíduos será idoso 2. Entre as Unidades Federativas brasileiras, a maior expectativa de vida encontrada foi em Santa Catarina (79,9 anos) e a menor no Maranhão (71,4 anos). Já em relação à proporção de idosos, nota-se que a região do Brasil com maior representatividade é a Sudeste com 46,2\%, seguida de Nordeste (26,5\%), Sul (15,9\%), Centro-oeste (6,15\%) e Norte (4,6\%) 3. Apesar da notável conquista referente ao aumento da expectativa de vida, observa-se que muitos desses idosos envelhecem com incapacidade, pois, conforme fundamentado na declaração da Década do Envelhecimento Saudável Entre os Anos 2020-2030 4, esses anos mais longos não consideram as mudanças em níveis de morbidades ou outros indicadores de condições de saúde 5 .

Observa-se que a população idosa tende a permanecer a maior parte do seu tempo diário em comportamento sedentário, como evidenciado no estudo de Malaquias et al. 6, que mostrou que pessoas com idade menor que 35 anos passam menos tempo em comportamento sedentário, enquanto aquelas com mais de 64 anos estão expostas a tempos prolongados em comportamento sedentário. Ademais, cerca de $80 \%$ do tempo acordado em idosos são despendidos em atividades sedentárias ( 8 a 12 horas por dia) 7,8,9,10,11,12,13,14. O comportamento sedentário engloba as atividades realizadas em vigília com gasto energético muito baixo, inferior a 1,5 equivalentes metabólicos, ou seja, equivalente à energia suficiente para se manter em repouso em termos de consumo de oxigênio de aproximadamente $3,5 \mathrm{~mL} / \mathrm{kg} / \mathrm{min} 15,16$. Existem diversas tipologias usadas para classificar o comportamento sedentário, as quais incluem, por exemplo, o tempo despendido em tarefas relacionadas ao transporte, leitura, alimentação e socialização. Além dessas, o "tempo de tela" tem sido o mais comumente analisado e associado a diferentes desfechos negativos de saúde 17,18,19, já que engloba qualquer tempo gasto em frente às telas de televisão, computador, celular e/ou tablet. Na última década, o tempo despendido em comportamento sedentário elevou 11,12 e os dados da Pesquisa Nacional de Saúde (PNS) de 2013 apresentaram que um terço da população adulta e idosa brasileira despende mais de 3 horas por dia em frente à televisão 20.

O tempo prolongado em comportamento sedentário está associado ao aumento na circunferência abdominal, baixa qualidade de vida e maior suscetibilidade à fragilidade e depressão 7,8,9,10,13,17. Recentemente, os maiores tempos em comportamento sedentário vêm sendo associados à presença de doenças agrupadas, como no caso da multimorbidade, que é a ocorrência simultânea de duas ou mais doenças. Além disso, indicadores socioeconômicos (como nível de escolaridade), estilo de vida (como consumo de álcool e inatividade física) e idade aumentada (entre 65-84 anos) também se apresentam como fatores de riscos para a presença de multimorbidade 21,22. Ressalta-se que, de acordo com os dados representativos de idosos brasileiros, a prevalência de multimorbidade foi superior à metade da população $(53,1 \%)$, e sabe-se que a multimorbidade tem sido associada à morte prematura, assim como à redução da expectativa de vida e maiores prevalências de incapacidade funcional 23 . No Brasil, um dos poucos estudos encontrados nessa temática utilizou os dados do inquérito Vigilância de Fatores de Risco e Proteção para Doenças Crônicas por Inquérito Telefônico (Vigitel) de 2013, e observou que a presença de doenças crônicas foi maior entre os idosos que passavam mais tempo em frente à televisão 24.

É relativamente incomum que idosos sejam afetados por somente uma doença crônica, pois determinadas doenças tendem a se acumular no mesmo indivíduo devido aos seus mecanismos fisiopatológicos e/ou fatores de risco semelhantes, o que induz a padrões de multimorbidade que sistematicamente e casualmente se agrupam $25,26,27$, tais como os padrões cardiopulmonar, vascularmetabólico e mental-musculoesquelético definidos por Rivera-Almaraz et al. 28. Algumas evidências demonstram que os idosos acometidos por padrões de multimorbidade apresentam maiores chances de incapacidade funcional 29,30, demência 25 , institucionalização 31 e mortalidade 32 .

Verifica-se que a relação entre comportamento sedentário e o acometimento por padrões de multimorbidade ainda é desconhecida na literatura e tal entendimento torna-se necessário devido à importância em integrar as necessidades complexas dos idosos, uma vez que os resultados de estudos com este objetivo poderão auxiliar na avaliação clínica de forma multidimensional no idoso. Além 
disso, servirá essencialmente para a proposição de ações e políticas direcionadas à promoção e prevenção na saúde que abordem mais especificamente o comportamento sedentário como potencial fator de risco para doenças crônicas, conforme preconizado pela Sociedade Americana de Geriatria 33, Política Nacional do Idoso 34 e recentes Diretrizes para Atividade Física e Comportamento Sedentário recomendadas pela Organização Mundial da Saúde (OMS) 35. Assim, este estudo teve como objetivo verificar a associação entre o comportamento sedentário, multimorbidade e seus padrões em idosos brasileiros.

\section{Métodos}

\section{Delineamento do estudo e população}

É um estudo transversal, adotando-se as recomendações do Strengthening the Reporting of Observational Studies in Epidemiology (STROBE) 36. Foram analisados os dados da população idosa brasileira ( $\geq 60$ anos) amostrada na PNS, realizada em 2019 pelo Instituto Brasileiro de Geografia e Estatística (IBGE) 3. O projeto da PNS 2019 foi aprovado pela Comissão Nacional de Ética em Pesquisa (CONEP), do Conselho Nacional de Saúde (CNS), sob o parecer no 3.529.376, emitido em agosto de 2019.

\section{Procedimentos de amostragem e coleta de dados}

As entrevistas foram realizadas com a utilização de dispositivos móveis de coleta, programados com o questionário da pesquisa. Inicialmente, o entrevistador fez contato com a pessoa responsável ou com algum dos moradores do domicílio selecionado. Foram identificados: o informante (que respondeu ao questionário domiciliar) e todos os moradores do domicílio, bem como o morador de 15 anos ou mais de idade (que responderia à entrevista individual), o qual foi selecionado por meio de programa de seleção aleatória nos dispositivos móveis de coleta. As entrevistas foram agendadas nas datas e horários mais convenientes para os informantes, prevendo-se duas ou mais visitas a cada domicílio. O banco disponibilizado pelo IBGE apresenta dados de 279.382 indivíduos adultos, e destes, 43.554 são idosos com 60 anos ou mais. Porém, a proporção amostrada variou de acordo com o indicador de interesse ${ }^{3}$.

A coleta de dados da PNS 2019 ocorreu entre os meses de agosto de 2019 e março de 2020. Maiores informações sobre a metodologia utilizada na PNS estão disponíveis em publicações prévias 36,37,38.

\section{Variável de exposição}

A variável de exposição foi o comportamento sedentário em tempo de tela 15. Essa variável foi obtida com o seguinte questionamento: "Em média, quantas horas por dia o(a) Sr.(a). costuma ficar assistindo à televisão (TV)?" e "Em um dia, quantas horas do seu tempo livre (excluindo o trabalho), o(a) Sr.(a). costuma usar computador, tablet ou celular para lazer, tais como: utilizar redes sociais, para ver notícias, vídeos, jogar, etc.?”. As opções de respostas incluíam: (1) menos de 1 hora; (2) 1 a menos de 2 horas; (3) 2 a menos de 3 horas; (4) 3 a menos de 6 horas; (5) Mais de 6 horas; e (6) Não realiza. Com base nas opções de respostas, o tempo despendido em comportamento sedentário foi categorizado e analisado no presente estudo em: (1) $<3$ horas; (2) 3 a 6 horas; e (3) $>6$ horas por dia, considerando-se as questões agrupadas do tempo gasto em frente à televisão e no computador, tablet ou celular (coletivamente chamado de "tempo de tela") 39.

\section{Desfecho do estudo}

Os desfechos foram a presença de multimorbidade (sim e não), considerada como o autorrelato da coexistência de duas ou mais doenças crônicas 40,41 (hipertensão arterial sistêmica - HAS; diabetes; hipercolesterolemia; doenças do coração, tais como infarto, angina, insuficiência cardíaca ou outros; e asma ou bronquite asmática; acidente vascular encefálico - AVE; asma, artrite ou reumatismo; problema crônico de coluna; depressão; doenças do pulmão, tais como enfisema pulmonar, bronquite 
crônica ou doença pulmonar obstrutiva crônica - DPOC; câncer; doenças osteomusculares relacionadas ao trabalho - DORT; e insuficiência renal crônica - IRC) e três padrões de multimorbidade (também categorizados em sim e não), considerando o autorrelato da ocorrência de duas ou mais doenças, anteriormente citadas, com características clínicas similares: (1) padrão cardiopulmonar: doença crônica no pulmão e doenças do coração; (2) padrão vascular-metabólico: HAS; diabetes; hipercolesterolemia; AVE; câncer e IRC; e (3) padrão mental-musculoesquelético: artrite ou reumatismo; problema crônico de coluna; DORT e depressão 28,42,43.

\section{Variáveis de ajuste}

As variáveis de ajustes foram analisadas em dois modelos separadamente. (1) variáveis sociodemográficas: sexo (feminino, masculino) 13, faixa etária (60-69, 70-79 e 80 anos ou mais) 44, escolaridade (sem escolaridade formal, 1-4 anos, 5-8, 9-11 e 12 ou mais anos) 44, estado civil (casado, divorciado/ solteiro, viúvo) 26; (2) variáveis de estilo de vida: etilismo (nenhum consumo de bebida alcoólica, < 1 vez por mês, > 1 vez por mês) 26; tabagismo (sim, não) 26, índice de massa corporal - IMC (baixo peso $<22 \mathrm{~kg} / \mathrm{m}^{2}$, peso adequado $22-27 \mathrm{~kg} / \mathrm{m}^{2}$, excesso de peso $>27 \mathrm{~kg} / \mathrm{m}^{2}$ ) 45 e nível de atividade física no lazer (suficiente ou insuficientemente ativos) 35. Para classificar os idosos em fisicamente ativos (> 150 minutos semanais) ou insuficientemente ativos (< 150 minutos semanais) no lazer, foram considerados os autorrelatos das práticas em atividades físicas vigorosas (corrida/cooper, corrida em esteira, ginástica aeróbica/spinning/step/jump, futebol, basquetebol ou tênis) e atividades físicas leves/ moderadas (caminhada, musculação, hidroginástica, ginástica/localizada/pilates/alongamento/ioga, natação, artes marciais/luta, bicicleta/bicicleta ergométrica, voleibol ou dança) 20,35,46,47.

\section{Análise dos dados}

Foi utilizado o programa estatístico Stata versão 15.0 (https://www.stata.com). Foram realizadas análises descritivas para todas as variáveis, com cálculo das prevalências e os respectivos intervalos de 95\% de confiança (IC95\%). Para testar as associações entre o comportamento sedentário com a presença de multimorbidade e dos seus padrões foram realizadas análises de regressão logística binária, estimando-se as odds ratio (OR) brutas e ajustadas e seus respectivos IC95\%. Os resultados com valor de $\mathrm{p} \leq$ 0,05 foram considerados estatisticamente significativos. Todas as análises consideraram o efeito do desenho do estudo, incorporando-se os pesos amostrais por meio do comando svy.

\section{Resultados}

Foram analisados os dados de 43.554 idosos, sendo a amostra composta predominantemente por mulheres (55,9\%) e com faixa etária entre 60 e 69 anos (55,6\%). Um terço dos idosos relatou ter estudado entre 1 e 4 anos e metade era casado. Em relação às características de estilo de vida, a maior parte da amostra disse ser insuficientemente ativo (81,3\%), não fumar $(88,2 \%)$ e não consumir bebida alcoólica (75,2\%). Menos da metade dos idosos (43,8\%) estava com peso adequado, e 40,6\% tinham excesso de peso (Tabela 1). Idosos que relataram comportamento sedentário de até 3 horas/dia representaram $68,8 \%$ da amostra, 20,1\% relataram entre 3 e 6 horas/dia e $11 \%$ mais de 6 horas/dia.

A prevalência de multimorbidade foi de 55,4\% (IC95\%: 54,6; 56,1). Em relação aos padrões de multimorbidade, observaram-se prevalências de 2,23\% (IC95\%: 2,35; 2,46) para o padrão cardiopulmonar, 13,6\% (IC95\%: 13,1; 14,1) para o padrão mental-musculoesquelético e 34,2\% (IC95\%: 33,5; 3,49) para o padrão vascular-metabólico. 
Tabela 1

Características sociodemográficas e de estilo de vida dos idosos brasileiros conforme a multimorbidade e os padrões de multimorbidade. Pesquisa Nacional de Saúde, Brasil, 2019.

\begin{tabular}{|c|c|c|c|c|c|}
\hline \multirow[t]{2}{*}{ Características } & \multirow[t]{2}{*}{$\mathrm{n}(\%)$ * } & \multirow{2}{*}{$\begin{array}{c}\text { Multimorbidade } \\
\text { [\% (IC95\%)] }\end{array}$} & \multicolumn{3}{|c|}{ Padrões de multimorbidade [\% (IC95\%)] } \\
\hline & & & Cardiopulmonar & $\begin{array}{c}\text { Mental- } \\
\text {-musculoesquelético }\end{array}$ & $\begin{array}{c}\text { Vascular- } \\
\text {-metabólico }\end{array}$ \\
\hline \multicolumn{6}{|c|}{ Faixa etária (anos) [n = 43.554] } \\
\hline $60-69$ & $24.247(55,6)$ & $52,2(51,3 ; 53,2)$ & $1,91(1,67 ; 2,20)$ & $13,9(13,2 ; 14,5)$ & $31,0(30,1 ; 31,8)$ \\
\hline $70-79$ & $13.209(30,3)$ & $59,4(58,1 ; 60,7)$ & $2,48(2,11 ; 2,91)$ & $13,6(12,8 ; 14,5)$ & $39,2(38,0 ; 40,4)$ \\
\hline$\geq 80$ & $6.098(14,0)$ & $58,9(57,0 ; 60,8)$ & $3,00(2,36 ; 3,79)$ & $12,4(11,1 ; 13,7)$ & $36,0(34,1 ; 37,8)$ \\
\hline \multicolumn{6}{|l|}{ Sexo $[n=43.554]$} \\
\hline Masculino & $19.203(44,0)$ & $46,2(45,1 ; 47,3)$ & $2,00(1,72 ; 2,32)$ & $6,42(5,92 ; 6,96)$ & $28,0(27,0 ; 29,0)$ \\
\hline Feminino & $24.351(55,9)$ & $62,5(61,6 ; 63,5)$ & $2,42(2,14 ; 2,74)$ & $19,4(18,6 ; 20,2)$ & $39,0(38,1 ; 40,0)$ \\
\hline \multicolumn{6}{|c|}{ Anos de estudos (anos) [ $n=42.312]$} \\
\hline Sem escolaridade & $10.616(25,0)$ & $54,9(53,4 ; 56,4)$ & $2,06(1,67 ; 2,54)$ & $11,5(10,7 ; 12,5)$ & $35,2(33,7 ; 36,7)$ \\
\hline $1-4$ & $15.196(35,9)$ & $58,1(56,9 ; 59,3)$ & $2,48(2,13 ; 2,88)$ & $14,7(13,9 ; 15,6)$ & $35,6(34,4 ; 36,7)$ \\
\hline $5-8$ & $5.305(12,5)$ & $53,8(51,7 ; 55,8)$ & $2,34(1,80 ; 3,04)$ & $14,2(12,9 ; 15,7)$ & $32,8(30,9 ; 34,7)$ \\
\hline $9-11$ & $7.128(16,8)$ & $53,7(51,9 ; 55,4)$ & $2,30(1,84 ; 2,89)$ & $14,6(13,3 ; 15,9)$ & $33,3(31,6 ; 34,9)$ \\
\hline 12 ou mais & $4.067(9,6)$ & $52,0(49,8 ; 54,3)$ & $1,59(1,14 ; 2,23)$ & $12,0(10,7 ; 13,5)$ & $32,0(30,0 ; 34,1)$ \\
\hline \multicolumn{6}{|l|}{ Estado civil [n = 43.554] } \\
\hline Casado & $22.226(51,0)$ & $53,6(52,6 ; 54,7)$ & $1,92(1,65 ; 2,24)$ & $12,0(11,3 ; 12,7)$ & $33,0(32,0 ; 34,0)$ \\
\hline Divorciado/Solteiro & $11.449(26,2)$ & $51,9(50,6 ; 53,2)$ & $2,20(18,4 ; 2,62)$ & $13,5(12,6 ; 14,4)$ & $31,3(30,1 ; 32,5)$ \\
\hline Viúvo & $9.879(22,6)$ & $61,8(60,5 ; 63,2)$ & $2,79(2,37 ; 3,28)$ & $16,4(15,4 ; 17,4)$ & $39,2(37,8 ; 40,5)$ \\
\hline \multicolumn{6}{|c|}{ Atividade física no lazer [ $n=22.585]$} \\
\hline Insuficientemente ativo & $18.374(81,3)$ & $56,1(55,3 ; 56,9)$ & $2,40(2,16 ; 2,66)$ & $14,0(13,4 ; 14,5)$ & $34,6(33,8 ; 35,4)$ \\
\hline Suficientemente ativo & $4.211(18,6)$ & $52,4(50,8 ; 54,0)$ & $1,60(1,23 ; 2,08)$ & $11,9(10,9 ; 13,0)$ & $32,8(31,3 ; 34,4)$ \\
\hline \multicolumn{6}{|l|}{ Tabagismo [n = 22.728] } \\
\hline Não & $20.048(88.2)$ & $56,4(55,6 ; 57,2)$ & $2,16(1,94 ; 2,39)$ & $13,7(13,2 ; 14,3)$ & $35,6(34,8 ; 36,3)$ \\
\hline Sim & $2.680(11,7)$ & $46,9(44,8 ; 49,0)$ & $2,82(2,18 ; 3,65)$ & $12,5(11,2 ; 13,9)$ & $23,1(21,3 ; 25,0)$ \\
\hline \multicolumn{6}{|l|}{ Etilismo $[\mathrm{n}=22.728]$} \\
\hline Não consome (vez/mês) & $17.096(75,2)$ & $57,7(56,8 ; 58,5)$ & $2,36(2,12 ; 2,63)$ & $14,7(14,1 ; 15,3)$ & $36,1(35,3 ; 36,9)$ \\
\hline$<1$ & $1.877(8,2)$ & $52,5(50,1 ; 55,0)$ & $2,25(1,60 ; 3,18)$ & $14,1(14,5 ; 15,9)$ & $30,2(28,0 ; 32,6)$ \\
\hline$>1$ & $3.755(16,5)$ & $47,2(45,5 ; 49,0)$ & $1,71(1,32 ; 2,20)$ & $8,92(8,00 ; 9,94)$ & $28,3(26,7 ; 29,8)$ \\
\hline \multicolumn{6}{|l|}{$\mathrm{IMC}[\mathrm{n}=22.726]$} \\
\hline Baixo peso & $3.519(15,4)$ & $46,9(45,1 ; 48,7)$ & $2,63(2,10 ; 3,29)$ & $1,16(10,5 ; 12,8)$ & $24,3(22,8 ; 25,9)$ \\
\hline Peso adequado & $9.971(43,8)$ & $51,2(50,1 ; 52,3)$ & $1,86(1,59 ; 2,18)$ & $11,9(11,2 ; 12,6)$ & $30,7(29,7 ; 31,7)$ \\
\hline Excesso de peso & $9.236(40,6)$ & $62,6(61,5 ; 63,7)$ & $2,49(2,16 ; 2,86)$ & $16,1(15,3 ; 16,9)$ & $41,2(40,1 ; 42,3)$ \\
\hline
\end{tabular}

IC95\%: intervalo de 95\% de confiança; IMC: índice de massa corporal.

* \% ponderado da categoria.

Na Figura 1, são apresentadas as prevalências de multimorbidade e seus padrões conforme as categorias do comportamento sedentário. A prevalência de multimorbidade foi maior no tempo superior a 6 horas por dia, com 64,6\%, da mesma forma para o padrão cardiopulmonar (3,5\%), padrão mental-musculoesquelético (16\%) e padrão vascular-metabólico $(41,4 \%)$. Observou-se aumento na prevalência de todos os desfechos conforme o incremento no tempo de comportamento sedentário. Esse aumento foi de 13 pontos percentuais (p.p.) para a multimorbidade, 26p.p. para o padrão cardiopulmonar e 36p.p. no padrão vascular-metabólico, ao comparar aqueles que ficavam menos de 3 horas com os que ficavam 6 ou mais horas por dia em comportamento sedentário. 


\section{Figura 1}

Prevalência de multimorbidade e padrões de multimorbidade segundo o tempo em comportamento sedentário entre idosos. Pesquisa Nacional de Saúde, Brasil, 2019.

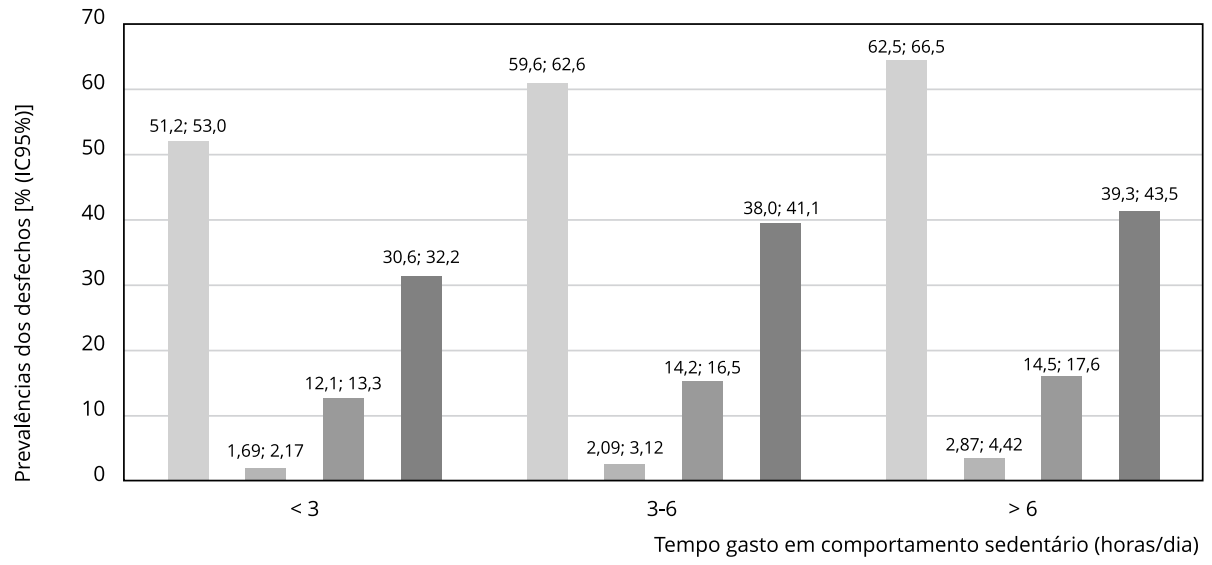

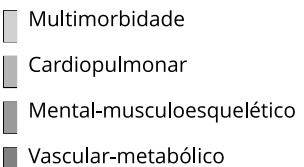

Vascular-metabólico

Nas Tabelas 2 e 3, estão apresentados os resultados das análises de regressão logística multivariável. Considerando o modelo final, ajustado para as variáveis sociodemográficas e de estilo de vida, verificaram-se associações positivas entre comportamento sedentário com a presença de multimorbidade e todos os padrões analisados. As chances de ter multimorbidade foram 39\% maiores (IC95\%: $1,29 ; 1,50)$ para os idosos que relataram permanecer entre 3 e 6 horas/dia em comportamento sedentário, e aumentaram para 58\% (IC95\%: 1,43; 1,74) naqueles com > 6 horas/dia, em comparação aos que permaneciam $<3$ horas/dia.

As chances de apresentar os padrões de multimorbidade também foram mais elevadas para os idosos que permaneceram maiores tempos em comportamento sedentário, sendo 73\% maiores (IC95\%: 1,33; 2,27) no padrão cardiopulmonar, 49\% (IC95\%: 1,35; 1,64) no padrão vascular-metabólico e $15 \%$ (IC95\%: 1,01; 1,31) no padrão mental-musculoesquelético para os idosos que ficavam mais de 6 horas/ dia em comportamento sedentário quando comparados aos que ficavam até 3 horas/dia. Já para os idosos que relataram permanecer de 3 a 6 horas/dia em comportamento sedentário, as chances de apresentar acometimento nos padrões vascular-metabólico e mental-musculoesquelético foram 39\% (IC95\%: 1,29; 1,50) e 11\% (IC95\%: 1,00; 1,24) mais elevadas, respectivamente, quando comparados aos que ficavam até 3 horas/dia em comportamento sedentário.

\section{Discussão}

A principal evidência deste estudo foi a de que os idosos que despendem mais de 3 horas/dia em comportamento sedentário tiveram chances significativamente maiores de apresentar multimorbidade, assim como todos os seus padrões, quando comparados àqueles que ficavam menos de 3 horas neste comportamento. Ressalta-se que os idosos que permaneciam tempos superiores a 6 horas por dia em comportamento sedentário apresentaram as chances mais elevadas de acometimento por todos os desfechos analisados.

No presente estudo, o comportamento sedentário em até 3 horas/dia foi observado em aproximadamente 7 a cada 10 idosos. Dados similares aos do presente trabalho em relação à prevalência da multimorbidade foram encontrados por Violan et al. 43, Nunes et al. 22 e Melo \& Lima 23, em que se mostra um fator preocupante, já que está associada aos maiores gastos em saúde 48 . Ademais, o padrão 
Tabela 2

Análises bruta e ajustada da regressão logística multivariada entre o comportamento sedentário e os padrões de multimorbidade em idosos brasileiros. Pesquisa Nacional de Saúde, Brasil, 2019.

\begin{tabular}{|c|c|c|c|c|c|c|c|c|c|}
\hline \multirow{2}{*}{$\begin{array}{l}\text { Comporta- } \\
\text { mento } \\
\text { sedentário } \\
\text { (horas/dia) }\end{array}$} & \multicolumn{3}{|c|}{ Cardiopulmonar } & \multicolumn{3}{|c|}{ Vascular-metabólico } & \multicolumn{3}{|c|}{ Mental-musculoesquelético } \\
\hline & $\begin{array}{c}\text { Bruta } \\
\text { OR } \\
\text { (IC95\%) }\end{array}$ & $\begin{array}{c}\text { Modelo } 1 \\
\text { OR } \\
\text { (IC95\%) }\end{array}$ & $\begin{array}{c}\text { Modelo } 2 \\
\text { OR } \\
\text { (IC95\%) }\end{array}$ & $\begin{array}{c}\text { Bruta } \\
\text { OR } \\
\text { (IC95\%) }\end{array}$ & $\begin{array}{c}\text { Modelo } 1 \\
\text { OR } \\
\text { (IC95\%) }\end{array}$ & $\begin{array}{c}\text { Modelo } 2 \\
\text { OR } \\
\text { (IC95\%) }\end{array}$ & $\begin{array}{c}\text { Bruta } \\
\text { OR } \\
\text { (IC95\%) }\end{array}$ & $\begin{array}{c}\text { Modelo } 1 \\
\text { OR } \\
\text { (IC95\%) }\end{array}$ & $\begin{array}{c}\text { Modelo } 2 \\
\text { OR } \\
\text { (IC95\%) }\end{array}$ \\
\hline$<3$ & 1,00 & 1,00 & 1,00 & 1,00 & 1,00 & 1,00 & 1,00 & 1,00 & 1,00 \\
\hline $3-6$ & $\begin{array}{c}1,34 \\
(1,05 ; 1,70)\end{array}$ & $\begin{array}{c}1,29 \\
(1,01 ; 1,66)\end{array}$ & $\begin{array}{c}1,27 \\
(0,99 ; 1,64)\end{array}$ & $\begin{array}{c}1,24 \\
(1,12 ; 1,37)\end{array}$ & $\begin{array}{c}1,40 \\
(1,30 ; 1,51)\end{array}$ & $\begin{array}{c}1,39 \\
(1,29 ; 1,50)\end{array}$ & $\begin{array}{c}1,42 \\
(1,32 ; 1,53)\end{array}$ & $\begin{array}{c}1,12 \\
(1,01 ; 1,24)\end{array}$ & $\begin{array}{c}1,11 \\
(1,00 ; 1,24)\end{array}$ \\
\hline$>6$ & $\begin{array}{c}1,88 \\
(1,46 ; 2,43)\end{array}$ & $\begin{array}{c}1,82 \\
(1,40 ; 2,38)\end{array}$ & $\begin{array}{c}1,73 \\
(1,33 ; 2,27)\end{array}$ & $\begin{array}{c}1,30 \\
(1,15 ; 1,48)\end{array}$ & $\begin{array}{c}1,50 \\
(1,36 ; 1,65)\end{array}$ & $\begin{array}{c}1,49 \\
(1,35 ; 1,64)\end{array}$ & $\begin{array}{c}1,54 \\
(1,40 ; 1,69)\end{array}$ & $\begin{array}{c}1,18 \\
(1,03 ; 1,34)\end{array}$ & $\begin{array}{c}1,15 \\
(1,01 ; 1,31)\end{array}$ \\
\hline
\end{tabular}

IC95\%: intervalo de 95\% de confiança; Modelo 1: ajustado para características sociodemográficas; Modelo 2: ajustado para características sociodemográficas e estilo de vida; OR: odds ratio.

Nota: valores em negrito referentes a associação estatisticamente significativa.

Tabela 3

Análises bruta e ajustada da regressão logística multivariada entre o comportamento sedentário e a multimorbidade em idosos brasileiros. Pesquisa Nacional de Saúde, Brasil, 2019.

\begin{tabular}{|c|c|c|c|}
\hline \multirow[t]{4}{*}{ Comportamento sedentário (horas/dia) } & \multicolumn{3}{|c|}{ Multimorbidade } \\
\hline & Bruta & Modelo 1 & Modelo 2 \\
\hline & OR & OR & OR \\
\hline & (IC95\%) & (IC95\%) & (IC95\%) \\
\hline$<3$ & 1,00 & 1,00 & 1,00 \\
\hline 3-6 & $1,44(1,34 ; 1,55)$ & $1,40(1,30 ; 1,51)$ & $1,39(1,29 ; 1,50)$ \\
\hline$>6$ & $1,67(1,52 ; 1,83)$ & $1,62(1,47 ; 1,78)$ & $1,58(1,43 ; 1,74)$ \\
\hline
\end{tabular}

IC95\%: intervalo de 95\% de confiança; Modelo 1: ajustado para características sociodemográficas; Modelo 2: ajustado para características sociodemográficas e estilo de vida; OR: odds ratio.

Nota: valores em negrito referentes a associação estatisticamente significativa.

vascular-metabólico foi mais prevalente em comparação aos outros padrões investigados. Yao et al. 49 também encontraram o padrão vascular-metabólico (HAS, dislipidemia, diabetes e AVE) como o mais prevalente em idosos chineses. As associações fisiopatológicas desse padrão podem ser explicadas pelo mecanismo dos distúrbios microvasculares (como retinopatia, nefropatia e neuropatia) e macrovasculares (como doença arterial coronariana, infarto do miocárdio, insuficiência cardíaca congestiva, AVE e doença vascular periférica), advindas das complicações promovidas pela diabetes e doenças cardíacas 50 .

As associações entre comportamento sedentário e multimorbidade verificadas neste trabalho foram similares às observadas por Christofoletti et al. 24, com maiores riscos de apresentar multimorbidade conforme o aumento do tempo em comportamento sedentário. Vancampfort et al. 21 avaliaram 34.129 adultos com idades $\geq 50$ anos em seis países de baixa a média renda e relataram que a multimorbidade foi associada às maiores chances (OR =1,41; IC95\%: 1,19; 1,66) de apresentarem comportamento sedentário mais elevado ( $\geq 8$ horas/dia), sugerindo que indivíduos com multimorbidade que vivem em países com condições socioeconômicas mais baixas são mais sedentários. Loprinzi 51 
verificou que para cada aumento de 60 minutos por dia em comportamento sedentário, os idosos apresentavam índice de multimorbidade mais alto e chances elevadas de apresentar multimorbidade. A associação entre multimorbidade e comportamento sedentário pode ser justificada pelo fato de o comportamento sedentário contribuir para a redução da capacidade aeróbica, força muscular, função metabólica e controle glicêmico. Além disso, o comportamento sedentário elevado pode propiciar mudanças estruturais nas artérias e na função endotelial, ocasionando múltiplos processos inflamatórios, menor sensibilidade à insulina, encurtamento dos telômeros e alteração na função cognitiva 52,53,54,55. Todas essas alterações podem levar à perda da homeostase do corpo e sistemas, aumentando as possibilidades de aparecimento das doenças 56.

Em relação à associação entre o comportamento sedentário e a presença dos padrões de multimorbidade, verificaram-se maiores chances de acometimento no padrão vascular-metabólico para os idosos que permaneciam entre 3 e 6 horas por dia em comportamento sedentário. Schmid \& Leitzmann 57 evidenciaram que o aumento de 2 horas/dia em comportamento sedentário foi relacionado a um aumento significativo no risco de câncer e esclarecem que o comportamento sedentário causa diminuição do gasto energético acompanhado de ganho de peso, facilitando a carcinogênese por diversas vias, dentre as quais destaca-se a resistência à insulina. Wilmot et al. 58 descrevem a relação entre o tempo sedentário com o aumento na incidência de diabetes e doenças cardiovasculares. Além disso, Bergouignan et al. 59 evidenciaram que o comportamento sedentário causa a desregulação metabólica por meio de quatro princípios: (1) resistência à insulina, (2) tráfego prejudicado de lipídios e hiperlipidemia, (3) mudança no uso do substrato para a glicose; e (4) mudança no tipo de fibra muscular e armazenamento de gordura ectópica. Nieste et al. 60 evidenciaram que reduzir o tempo sedentário em 1 hora/dia diminui os marcadores cardiometabólicos de risco à saúde, como circunferência da cintura, porcentagem de gordura e controle glicêmico. Dessa forma, aumentar o nível de atividade física e reduzir o comportamento sedentário são modificações eficazes para reduzir o risco de doenças nesse tipo de padrão, propiciando melhorias na ação da insulina, pressão arterial, dislipidemia e também melhorando a aptidão cardiorrespiratória e a longevidade 27,51,61.

No presente estudo, os idosos que relataram permanecer 6 ou mais horas por dia em comportamento sedentário tiveram maiores chances de acometimento no padrão cardiopulmonar. A pesquisa de Vaz Fragoso et al. 62 esclareceu que o estado sedentário elevado esteve associado à dispneia e ao comprometimento respiratório (volume expiratório forçado em 1 segundo e pressão inspiratória máxima menor que o limite inferior normal). De modo semelhante, o estudo de Kaneko 63 mostrou que maiores tempos em comportamento sedentário contribuíram para a redução da capacidade vital forçada, na qual esta relação pode ser pela diminuição da mobilidade do tórax e do abdômen advinda do tempo excessivo em comportamento sedentário, reforçando-se que a função pulmonar é um importante preditor de mortalidade cardiovascular. Thyfault et al. 53 apontaram que a interrupção do comportamento sedentário com exercício físico protege contra diminuições no volume sistólico, atrofia cardíaca e força muscular. Considerando indivíduos com DPOC já instalado, Alyami et al. 64 evidenciaram que a maior parte daqueles com $>40$ anos permaneciam maiores períodos do dia em comportamento sedentário, quando comparados aos indivíduos saudáveis, sendo $25 \%$ maior o tempo em posições sentadas e $200 \%$ maior o tempo em posições deitadas 65 .

Diante do exposto, cabe ressaltar que a OMS atualizou, em 2020, suas recomendações para a diminuição do comportamento sedentário e o concomitante aumento da prática de atividade física regular entre idosos, enfatizando que a adoção de comportamentos mais ativos são capazes de proteger a presença de multimorbidade 35 . Foi recomendado que os idosos realizem semanalmente pelo menos 150 a 300 minutos de atividades físicas aeróbicas de moderada intensidade ou 75 a 150 minutos de atividades físicas aeróbicas de vigorosa intensidade. No entanto, essas diretrizes não oferecem recomendações sobre a quantidade de horas a serem consideradas e/ou evitadas no tempo sentado.

Apesar dos achados relevantes encontrados para os idosos brasileiros amostrados, cabe destacar algumas limitações do presente estudo, tais como o uso de medidas autorreferidas para avaliação do comportamento sedentário, as quais podem apresentar viés de informação pelo entrevistado, com a possibilidade de sub ou superestimação do tempo em comportamento sedentário 66. Apesar disso, vale lembrar que a avaliação subjetiva tende a ser mais fácil e com baixo custo, podendo ser empregada tanto em pesquisas epidemiológicas quanto nas avaliações de idosos na prática clínica. Ainda que o tempo gasto em frente às telas seja o mais comumente tipo de comportamento sedentário relatado 
pelos idosos 67, a ausência da avaliação de outras tipologias, assim como de outras categorias distintas de 3 horas/dia na classificação do comportamento sedentário 14 pela PNS, dificultou a comparação fidedigna dos nossos resultados com outros estudos brasileiros semelhantes.

Um ponto que merece ser destacado é que, com o estabelecimento do estado de pandemia em 2020 pela OMS devido ao novo coronavírus (COVID-19), o comportamento sedentário aumentou consideravelmente em idosos 12,68. Sabe-se que a presença de padrões de multimorbidade complica as pessoas infectadas 69, sendo, portanto, mais um motivo importante para buscar medidas preventivas contra as doenças crônicas, como a redução do tempo em comportamento sedentário em idosos. Além disso, conscientizar a substituição do comportamento sedentário por atividade física pelo tempo recomendado ou ocupação do tempo sentado por tarefas cognitivamente mais estimulantes (como ler ou jogar jogos de memória e de tabuleiro) 70 .

\section{Conclusão}

Verificaram-se que os idosos que despendiam mais de 3 horas/dia em comportamento sedentário tiveram maiores chances de apresentar multimorbidade, assim como todos os seus padrões, quando comparados àqueles que ficavam até 3 horas neste comportamento. As chances de multimorbidade e seus diferentes padrões foram ainda maiores para os idosos que ficavam mais de 6 horas/dia em comportamento sedentário. Assim, acredita-se que os nossos resultados mostram a relevância de instituir programas com o objetivo de reduzir e/ou interromper o comportamento sedentário nos idosos brasileiros, uma vez que a presença de múltiplas doenças crônicas no mesmo indivíduo aumenta a sua necessidade de utilização de cuidados com a saúde e o seu risco de morte precoce. Além disso, políticas voltadas à modificação de fatores de risco comportamentais para a ocorrência de multimorbidade nessa população poderão refletir não somente na melhor qualidade de vida durante a longevidade, mas também na redução dos custos gerados pelo sistema público de saúde, já que com elas, espera-se alcançar a diminuição por consultas com profissionais de saúde, assistências ambulatoriais e hospitalizações. 


\section{Colaboradores}

L. M. Cândido e A. L. Danielewicz contribuíram na concepção do estudo, planejamento, análise, interpretação dos dados, revisão bibliográfica, escrita e revisão do artigo. K. J. P. Wagner contribuiu no planejamento, análise, interpretação dos dados, escrita e revisão do artigo. M. E. Costa, E. Pavesi e N. C. P. Avelar contribuíram na revisão bibliográfica, escrita e revisão do artigo.

\section{Informações adicionais}

ORCID: Letícia Martins Cândido (0000-00023564-5322); Kátia Jakovljevic Pudla Wagner (0000-0002-3649-3121); Maria Eduarda da Costa (0000-0002-3026-5072); Eloísa Pavesi (0000-00030428-6694); Núbia Carelli Pereira de Avelar (00000003-4212-4039); Ana Lúcia Danielewicz (00000003-1563-0470).

\section{Referências}

1. Instituto Brasileiro de Geografia e Estatística. Em 2019, expectativa de vida era de 76,6 anos. Agência IBGE de Notícias 2020; 26 nov. https://agenciadenoticias.ibge.gov.br/agenciasala-de-imprensa/2013-agencia-de-noticias/ releases/29502-em-2019-expectativa-de-vida -era-de-76-6-anos.

2. Population Division, Department of Economic and Social Affairs, United Nations. World population ageing 2019: highlights. https:// www.un.org/development/desa/pd/sites/ www.un.org.development.desa.pd/files/fi les/documents/2020/Jan/worldpopulationa geing2019-highlights.pdf (acessado em 20/ Jun/2020)

3. Instituto Brasileiro de Geografia e Estatística. Pesquisa Nacional de Saúde 2019: informações sobre domicílios, acesso e utilização dos serviços de saúde. Brasil, grandes regiões e unidades da federação. Rio de Janeiro: Instituto Brasileiro de Geografia e Estatística; 2020.

4. Organização Mundial da Saúde. Década do envelhecimento saudável 2020-2030. https:// sbgg.org.br/wp-content/uploads/2020/10/ D\%C3\%A9cada-do-Envelhecimento-Saud \%C3\%A1vel-2020-2030.pdf (acessado em Set/2021).

5. Guimarães RM, Andrade FCD. Expectativa de vida com e sem multimorbidade entre idosos brasileiros: Pesquisa Nacional de Saúde 2013. Rev Bras Estud Popul 2020; 37:e0117.

6. Malaquias BSS, Fonseca LRM, Sousa MC, Cornélio MPM, Corrêa TB, Martins MM, et al. Determinantes do comportamento sedentário ao longo da vida. Arquivos de Ciências do Esporte 2019; 6:50-5.

7. Arins GCB, Santos CES, Giehl MWC, Benedetti TRB, Oliveira C, D'Orsi E, et al. Neighborhood environmental characteristics and sedentary behavior in later life: the EpiFloripa Study. Rev Bras Ativ Fís Saúde 2019; 23:1-7.

8. Chen S, Chen T, Kishimoto H, Yatsugi H, Kumagai S. Associations of objectively measured patterns of sedentary behavior and physical activity with frailty status screened by The Frail Scale in Japanese Community-Dwelling Older Adults. J Sports Sci Med 2020; 19:166-74.

9. Hadgraft NT, Winkler E, Climie RE, Grace MS, Romero L, Owen N, et al. Effects of sedentary behaviour interventions on biomarkers of cardiometabolic risk in adults: systematic review with meta-analyses. Br J Sports Med 2021; 55:144-54.

10. Dontje ML, Leask CF, Harvey J, Skelton DA, Chastin SFM. Why older adults spend time sedentary and break their sedentary behavior: a mixed-methods approach using life-logging equipment. J Aging Phys Act 2018; 26:259-66.

11. LeBlanc AG, Gunnell KE, Prince SA, Saunders TJ, Barnes JD, Chaput J-P. The Ubiquity of the screen: an overview of the risks and benefits os screen time in our modern world. Translational Journal of the ACSM 2017; 2:104-13. 
12. Malta DC, Szwarcwald CL, Barros MBA, Gomes CS, Machado IE, Souza Júnior PRB, et al. A pandemia da COVID-19 e as mudanças no estilo de vida dos brasileiros adultos: um estudo transversal, 2020. Epidemiol Serv Saúde 2020; 29:e2020407.

13. Prince SA, Cardilli L, Reed JL, Saunders TJ, Kite C, Douillette K, et al. A comparison of self-reported and device measured sedentary behaviour in adults: a systematic review and meta-analysis. Int J Behav Nutr Phys Act 2020; $17: 31$.

14. Rezende L, Rodrigues Lopes M, Rey-López J, Matsudo V, Luiz OC. Sedentary behavior and health outcomes: an overview of systematic reviews. PLoS One 2014; 9:e105620.

15. Tremblay MS, Aubert S, Barnes JD, Saunders TJ, Carson V, Latimer-Cheung AE, et al. Sedentary Behavior Research Network (SBRN) Terminology Consensus Project process and outcome. Int J Behav Nutr Phys Act 2017; 14:75.

16. Faria CRC, Melo FCL, Ravagnani FCP, Homero F, Burini P. Estimation of the metabolic equivalent (met) of an exercise protocol based on indirect calorimetry. Rev Bras Med Esporte 2013; 19:134-8.

17. Compernolle S, Bourdeaudhuij I, Cardon G, van Dyck D. Sex-specific typologies of older adults' sedentary behaviors and their associations with health-related and socio-demographic factors: a latent profile analysis. BMC Geriatr 2021; 21:66.

18. Wu L, Sun S, He Y, Jiang B. The effect of interventions targeting screen time reduction: a systematic review and meta-analysis. Medicine (Baltimore) 2016; 95:e4029.

19. Banks E, Jorm L, Rogers K, Clements M, Bauman A. Screen-time, obesity, ageing and disability: findings from 91266 participants in the 45 and Up Study. Public Health Nutr 2011; 14:34-43.

20. Mielke GI, Malta DC, Sá GB, Reis RS, Hallal PC. Regional differences and correlates of leisure time physical activity in Brazil: results from the Brazilian National Health Survey-2013. Rev Bras Epidemiol 2015; 18:158-69.

21. Vancampfort D, Stubbs B, Koyanagi A. Physical chronic conditions, multimorbidity and sedentary behavior amongst middle-aged and older adults in six low- and middle-income countries. Int J Behav Nutr Phys Act 2017; 14:147.

22. Nunes B, Batista S, Andrade F, Souza Junior P, Lima-Costa M, Facchini L. Multimorbidity: the Brazilian Longitudinal Study of Aging (ELSI-Brazil). Rev Saúde Pública 2018; 52 Suppl 2:10s.

23. Melo LA, Lima KC. Prevalência e fatores associados a multimorbidades em idosos brasileiros. Ciênc Saúde Colet 2020; 25:3869-77.

24. Christofoletti M, Del Duca GF, Umpierre D, Malta DC. Chronic noncommunicable diseases multimorbidity and its association with physical activity and television time in a representative Brazilian population. Cad Saúde Pública 2019; 35:e00016319.
25. Grande G, Marengoni A, Vetrano D, RosoLlorach A, Rizzuto D, Zucchelli A, et al. Multimorbidity burden and dementia risk in older adults: the role of inflammation and genetics. Alzheimers Dement 2021; 17:768-76

26. Marengoni A, Roso-Llorach A, Vetrano DL, Fernández-Bertolín S, Guisado-Clavero M, Violán C, et al. Patterns of multimorbidity in a population-based cohort of older people: sociodemographic, Lifestyle, clinical, and functional differences. J Gerontol A Biol Sci Med Sci 2020; 75:798-805.

27. Prados-Torres A, Calderón-Larrañaga A, Hancco-Saavedra J, Poblador-Plou B, van den Akker M. Multimorbidity patterns: a systematic review. J Clin Epidemiol 2014; 67:254-66.

28. Rivera-Almaraz A, Manrique-Espinoza B, Ávila-Funes JA, Chatterji S, Naidoo N, Kowal $\mathrm{P}$, et al. Disability, quality of life and all-cause mortality in older Mexican adults: association with multimorbidity and frailty. BMC Geriatr 2018; 18:236.

29. Vetrano DL, Rizzuto D, Calderón-Larrañaga A, Onder G, Welmer AK, Qiu C, et al. Walking speed drives the prognosis of older adults with cardiovascular and neuropsychiatric multimorbidity. Am J Med 2019; 132:1207-15.e6.

30. Schmidt TP, Wagner KJP, Schneider IJC, Danielewicz AL. Padrões de multimorbidade e incapacidade funcional em idosos brasileiros: estudo transversal com dados da Pesquisa Nacional de Saúde. Cad Saúde Pública 2020; 36:e0241619.

31. Marengoni A, Akugizibwe R, Vetrano DL, Roso-Llorach A, Onder G, Welmer A-K, et al. Patterns of multimorbidity and risk of disability in community-dwelling older persons. Aging Clin Exp Res 2021; 33:457-62.

32. Vetrano DL, Roso-Llorach A, Fernández S, Guisado-Clavero M, Vilán C, Onder G, et al. Twelve-year clinical trajectories of multimorbidity in a population of older adults. Nature Communications 2020; 11:3223.

33. Guiding principles for the care of older adults with multimorbidity: an approach for clinicians: American Geriatrics Society expert panel on the care of older adults with multimorbidity. J Am Geriatr Soc 2012; 60:E1-25.

34. Brasil. Lei no 8.842, de 4 de Janeiro de 1994. Dispõe sobre a política nacional do idoso, cria o Conselho Nacional do Idoso e dá outras providências. Ministério da Previdência e Assistência Social. Diário Oficial da União 1994; 5 jan.

35. World Health Organization. Guidelines on physical activity and sedentary behaviour. Geneva: World Health Organization; 2020.

36. von Elm E, Altman D, Egger M, Pocock S, Gøtzsche P, Vandenbroucke J. The Strengthening the Reporting of Observational Studies in Epidemiology (STROBE) statement: guidelines for reporting observational studies. J Clin Epidemiol 2008; 61:344-9.

37. Stopa SR, Szwarcwald CL, Oliveira MM, Gouvea ECDP, Vieira MLFP, Freitas MPS, et al. National Health Survey 2019: history, methods and perspectives. Epidemiol Serv Saúde 2020; 29:e2020315. 
38. Szwarcwald CL, Malta DC, Pereira CA, Vieira ML, Conde WL, Souza Júnior PR, et al. National Health Survey in Brazil: design and methodology of application. Ciên Saúde Colet 2014; 19:333-42.

39. Departamento de Análise em Saúde e Vigilância de Doenças não Transmissíveis, Secretaria de Vigilância em Saúde, Ministério da Saúde. Vigitel Brasil 2019: vigilância de fatores de risco e proteção para doenças crônicas por inquérito telefônico: estimativas sobre frequência e distribuição sociodemográfica de fatores de risco e proteção para doenças crônicas nas capitais dos 26 estados brasileiros e no Distrito Federal em 2019. Brasília: Ministério da Saúde; 2020.

40. Johnston MC, Crilly M, Black C, Prescott GJ, Mercer SW. Defining and measuring multimorbidity: a systematic review of systematic reviews. Eur J Public Health 2019; 29:182-9.

41. Marengoni A, Angleman S, Melis R, Mangialasche F, Karp A, Garmen A, et al. Aging with multimorbidity: a systematic review of the literature. Ageing Res Rev 2011; 10:430-9.

42. Garin N, Koyanagi A, Chatterji S, Tyrovolas S, Olaya B, Leonardi M, et al. Global multimorbidity patterns: a cross-sectional, populationbased, multi-country study. J Gerontol A Biol Sci Med Sci 2016; 71:205-14.

43. Violan C, Foguet-Boreu Q, Flores-Mateo G, Salisbury C, Blom J, Freitag M, et al. Prevalence, determinants and patterns of multimorbidity in primary care: a systematic review of observational studies. PLoS One 2014; 9:e102149.

44. Schäfer I, von Leitner EC, Schön G, Koller D, Hansen H, Kolonko T, et al. Multimorbidity patterns in the elderly: a new approach of disease clustering identifies complex interrelations between chronic conditions. PLoS One 2010; 5:e15941.

45. Akugizibwe R, Calderón-Larrañaga A, RosoLlorach A, Onder G, Marengoni A, Zucchelli $\mathrm{A}$, et al. Multimorbidity patterns and unplanned hospitalisation in a cohort of older adults. J Clin Med 2020; 9:4001.

46. Haskell WL, Lee IM, Pate RR, Powell KE, Blair SN, Franklin BA, et al. Physical activity and public health: updated recommendation for adults from the American College of Sports Medicine and the American Heart Association. Circulation 2007; 116:1081-93.

47. Schäfer I, Hansen H, Schön G, Höfels S, Altiner A, Dahlhaus A, et al. The influence of age, gender and socio-economic status on multimorbidity patterns in primary care. First results from the multicare cohort study. BMC Health Serv Res 2012; 12:89.

48. Ha N, Le N, Khanal V, Moorin R. Multimorbidity and its social determinants among older people in southern provinces, Vietnam. Int J Equity Health 2015; 14:50.
49. Yao SS, Cao GY, Han L, Chen ZS, Huang ZT, Gong P, et al. Prevalence and patterns of multimorbidity in a nationally representative sample of older chinese: results from the China Health and Retirement Longitudinal Study. J Gerontol A Biol Sci Med Sci 2020; 75:1974-80.

50. Long AN, Dagogo-Jack S. Comorbidities of diabetes and hypertension: mechanisms and approach to target organ protection. J Clin Hypertens (Greenwich) 2011; 13:244-51.

51. Loprinzi PD. Sedentary behavior and medical multimorbidity. Physiol Behav 2015; 151:395-7.

52. Guerra PH, Mielke GI, Garcia LMT. Comportamento sedentário. Corpoconsciência 2014; 18:23-36.

53. Thyfault JP, Du M, Kraus WE, Levine JA, Booth FW. Physiology of sedentary behavior and its relationship to health outcomes. Med Sci Sports Exerc 2015; 47:1301-5.

54. Lozado YA, Barbosa RS, Caires SS, Bomfim BSM, Santos L. Implicaciones del alto comportamiento sedentario para la salud de los ancianos: una revisón de literatura. Práticas e Cuidado: Revista de Saúde Coletiva 2020; 1:e9994.

55. Olanrewaju O, Stockwell S, Stubbs B, Smith L. Sedentary behaviours, cognitive function, and possible mechanisms in older adults: a systematic review. Aging Clin Exp Res 2020; 32:969-84.

56. Booth FW, Roberts CK, Laye MJ. Lack of exercise is a major cause of chronic diseases. Compr Physiol 2012; 2:1143-211.

57. Schmid D, Leitzmann MF. Television viewing and time spent sedentary in relation to cancer risk: a meta-analysis. J Natl Cancer Inst 2014; 106:dju098.

58. Wilmot EG, Edwardson CL, Achana FA, Davies MJ, Gorely T, Gray LJ, et al. Sedentary time in adults and the association with diabetes, cardiovascular disease and death: systematic review and meta-analysis. Diabetologia 2012; 55:2895-905.

59. Bergouignan A, Rudwill F, Simon C, Blanc S. Physical inactivity as the culprit of metabolic inflexibility: evidence from bed-rest studies. J Appl Physiol (1985) 2011; 111:1201-10.

60. Nieste I, Franssen W, Spaas J, Bruckers L, Savelberg H, Eijnde B. Lifestyle interventions to reduce sedentary behaviour in clinical populations: a systematic review and meta-analysis of different strategies and effects on cardiometabolic health. Prev Med 2021; 148:106593.

61. Kirchberger I, Meisinger C, Heier M, Zimmermann AK, Thorand B, Autenrieth CS, et al. Patterns of multimorbidity in the aged population. Results from the KORA-Age study. PLoS One 2012; 7:e30556.

62. Vaz Fragoso C, Beavers D, Hankinson J, Flynn G, Berra K, Kritchevsky S, et al. Respiratory impairment and dyspnea and their associations with physical inactivity and mobility in sedentary community-dwelling older persons. J Am Geriatr Soc 2014; 62:622-8. 
63. Kaneko H. Association of respiratory function with physical performance, physical activity, and sedentary behavior in older adults. J Phys Ther Sci 2020; 32:92-7.

64. Alyami M, Jenkins S, Hill K. Walking-based activity and sedentary behavior in Saudi males with chronic obstructive pulmonary disease. Saudi Med J 2018; 39:506-13.

65. McKeough ZJ, Large SL, Spencer LM, Cheng SWM, McNamara RJ. An observational study of self-reported sedentary behaviour in people with chronic obstructive pulmonary disease and bronchiectasis. Braz J Phys Ther 2020; 24:399-406.

66. Atkin AJ, Gorely T, Clemes SA, Yates T, Edwardson C, Brage S, et al. Methods of measurement in epidemiology: sedentary behaviour. Int J Epidemiol 2012; 41:1460-71.
67. Mielke GI, Silva ICM, Owen N, Hallal PC. Brazilian adults' sedentary behaviors by life domain: population-based study. PLoS One 2014; 9:e91614.

68. Sepúlveda-Loyola W, Rodríguez-Sánchez I, Pérez-Rodríguez P, Ganz F, Torralba R, Oliveira DV, et al. Impact of social isolation due to COVID-19 on health in older people: mental and physical effects and recommendations. J Nutr Health Aging 2020; 24:938-47.

69. Fernández-Niño JA, Guerra-Gómez JA, Idrovo AJ. Multimorbidity patterns among COVID-19 deaths: proposal for the construction of etiological models. Rev Panam Salud Pública 2020; 44:e166.

70. Horacio PR, Avelar NCP, Danielewicz AL. Comportamento sedentário e declínio cognitivo em idosos comunitários. Rev Bras Ativ Fís Saúde 2021; 26:1-8. 


\section{Abstract}

Sedentary behavior emerges as an important determinant of health in elderly persons, but its relationship to multimorbidity and its patterns in the Brazilian population have received little attention in epidemiological studies. Such associations can assist the elaboration of public policies aimed at modification of this behavior. This study thus aimed to assess the association between sedentary behavior and multimorbidity and its patterns in elderly Brazilians. This was a cross-sectional study of 43,554 elderly Brazilians in the Brazilian National Health Survey, 2019. Self-reported $S B$ was categorized as < 3; 3-6; and > 6 hours a day. The presence of multimorbidity and its patterns were analyzed by self-report of two or more chronic diseases, where the three established patterns considered the diseases with similar clinical characteristics: (1) cardiopulmonary; (2) vascularmetabolic; and (3) mental-musculoskeletal. The associations were measured by binary logistic regression. Elderly persons that spent 3-6 hours/ day in sedentary behavior had lower odds $(O R=$ 1.39; 95\%CI: 1.29 ; 1.50$)$ of presenting multimorbidity, vascular-metabolic pattern $(O R=1.39$; 95\%CI: 1.29; 1.50), and mental-musculoskeletal pattern $(O R=1.11 ; 95 \%$ CI: 1.00; 1.24). Sedentary behavior $>6$ hours/day was associated with higher odds of multimorbidity $(\mathrm{OR}=1.58$; $95 \% \mathrm{CI}$ : $1.43 ; 1.74)$ and the cardiopulmonary $(O R=1.73$; 95\% CI: $1.33 ; 2.27)$, vascular-metabolic $(O R=$ 1.49; 95\%CI: 1.35; 1.64), and mental-musculoskeletal $(O R=1.15 ; 95 \% C I: 1.01 ; 1.31)$ patterns when compared to elders with 3 hours/day of sedentary behavior. This showed that extensive time in sedentary behavior is a relevant risk factor for multimorbidity and its patterns in the elderly.

Aged; Multimorbidity; Sedentary Behavior; Risk Factors

\section{Resumen}

El comportamiento sedentario emerge como un importante determinante de salud en personas ancianas, no obstante, su relación con la multimorbilidad y los patrones de enfrentamiento a la misma en estudios epidemiológicos se han investigado poco en la población brasileña. Tales asociaciones podrán apoyar en la elaboración de políticas públicas, con vistas a la modificación de ese comportamiento. Por ello, el objetivo de este estudio fue evaluar la asociación entre comportamiento sedentario con multimorbilidad y sus patrones en ancianos brasileños. Es un estudio transversal, con 43.554 ancianos de la Encuesta Nacional de Salud de 2019. El comportamiento sedentario autoinformado se categorizó en $<3 ; 3-6 ; y>6$ horas por día. La presencia de multimorbilidad y sus patrones se analizaron mediante el autoinforme de la coexistencia de dos o más enfermedades crónicas, siendo que los tres patrones establecidos consideraron las enfermedades con características clinicas similares: (1) cardiopulmonar; (2) vascularmetabólica y (3) mental-musculoesquelética. Las asociaciones fueron realizadas mediante regresión logistica binaria. Los ancianos que pasaban 3-6h/ dí en comportamiento sedentario tuvieron mayores oportunidades $(O R=1,39$; IC95\%: 1,29; 1,50) de presentar multimorbilidad, patrón vascularmetabólico (OR = 1,39; IC95\%: 1,29; 1,50) y mental-musculoesquelético (OR = 1,11; IC95\%: 1,00; 1,24). Cuando el comportamiento sedentario fue $>$ 6 horas/dia hubo mayores oportunidades de multimorbilidad (OR = 1,58; IC95\%: 1,43; 1,74), y de los patrones cardiopulmonar $(\mathrm{OR}=1,73$; IC95\%: $1,33 ; 2,27)$, vascular-metabólico $(O R=1,49$; IC95\%: 1,35; 1,64) y mental-musculoesquelético $(O R=1,15$; IC95\%: 1,01; 1,31), cuando se compara con aquellos que estaban hasta 3 horas/dia. De esa forma, se evidenció que tiempos elevados en comportamiento sedentario se mostraron factores de riesgo relevantes para la ocurrencia de multimorbilidad y sus patrones en ancianos.

Anciano; Multimorbilidad; Conducta Sedentaria; Factores de Riesgo
Recebido em 24/Mai/2021

Versão final reapresentada em 29/Jul/2021

Aprovado em 09/Ago/2021 